\title{
The importance of keratinized gingiva surrounding dental implants
}

\author{
Abstract \\ Purpose: The purpose of this study was to determine if keratinized gingiva has an effect \\ on the success of implants. \\ Materials and methods: Sixty nine implants were used in this study. The amount of \\ keratinized gingiva was measured and divided into two groups; less than $2 \mathrm{~mm}$ and greater \\ than $2 \mathrm{~mm}$. The amount of keratinized gingiva was compared to clinical parameters such as \\ bleeding upon probing, redness, and pocket depths to determine whether implant success \\ was related to the amount of keratinized gingiva.
}

Results: Chi square and regression analysis were used to analyze the data. All implants survived independent of the amount of keratinized gingiva. Patients with less than $2 \mathrm{~mm}$ of keratinized gingiva displayed increased bleeding upon probing and redness, which was statistically significant $(\mathrm{p}=0.023)$, indicating increased inflammation due to lack of keratinized gingiva.

Conclusion: Amount of keratinized gingiva did not affect the success rate of implants. However, implants with less than $2 \mathrm{~mm}$ of keratinized gingiva exhibited increased bleeding upon probing, redness, and inflammation, which may contribute to later failure.
Volume 2 Issue 5 - 2015

\author{
Pouran Famili, Anita Desai \\ Department of Periodontics and Preventive Dentistry, \\ University of Pittsburgh, USA
}

\begin{abstract}
Correspondence: Pouran Famili, Department of Periodontics and Preventive Dentistry, University of Pittsburgh, BI0I Salk Hall, 350 I Terrace Street, Pittsburgh PA I526I, USA, Tel 4I2
\end{abstract} 648-9997,Email prof@pitt.edu

Received: June 12, 2015 | Published: June 23, 2015

Keywords: keratinized gingiva, implants, inflammation, stability, dental implants

\section{Introduction}

There is a great deal of controversy in the literature about the importance of keratinized gingiva around dental implants and the amount, if any, required for implant health. Some studies conclude that peri-implant health could be maintained even in the absence of keratinized gingiva providing adequate oral hygiene is employed. ${ }^{1}$ Other studies suggest that areas of minimal keratinized gingiva have decreased tissue resistance allowing plaque accumulation, which increases the risk of gingival inflammation, marginal bone loss, and increased gingival recession. ${ }^{1}$ We hypothesize that implants surrounded by less than $2 \mathrm{~mm}$ of keratinized gingiva are more susceptible to failure due to decreased resistance of the tissues to bacterial infiltration, leading to increased tissue breakdown, increased probing depths surrounding the implants, increased bleeding upon probing, and increased bone loss. The purpose of this study is to determine whether a minimum width of $2 \mathrm{~mm}$ of keratinized gingiva around dental implants is necessary for the health and stability of the surrounding soft and hard tissues of the periodontium. Knowing this will help clinicians determine whether or not gingival augmentation to increase the amount of keratinized gingiva is required prior to implant therapy.

\section{Materials and methods}

A cross-sectional study was done to determine implant health/ success when looking specifically at the amount of keratinized gingiva surrounding the implant. Implant health, as we determined, was the absence of bleeding upon probing, redness and inflammation, suppuration, and mobility, pocket depths less than or equal to $3 \mathrm{~mm}$, and no radiographic evidence of progressive crestal bone loss.
Participants were randomly selected among patients presenting to the Graduate Periodontics Clinic or the Multidisciplinary Implant Center at the University of Pittsburgh school of Dental Medicine for routine maintenance appointments. Subjects were 21years of age or older, and required to have had an implant-supported restoration placed a minimum of six months prior. Two examiners, one resident and one faculty member in the Graduate Periodontics Department, recorded the data collected. Both examiners were calibrated and inter and intraexaminer reliability was evaluated.

The following data was recorded for each implant: number and site of the implant; width of keratinized gingiva; pocket depth; presence or absence of bleeding upon probing; presence or absence of suppuration; mobility of the implant; gingival color; radiographic bone level; time since implant placement; type of implant; smoking history in packs per year; age; and gender. The width of keratinized gingiva was measured at the mid facial aspect of each implant using a Michigan probe. Measurements taken from the muco gingival junction to the free gingival margin were measured to the nearest millimeter. Pocket depths were measured to the nearest millimeter using the Michigan probe at six surfaces of each implant: mesial-buccal, mid buccal, disto buccal, mesial-lingual, mid lingual, disto lingual. Radiographic bone level was measured from a fixed reference point to the alveolar crest on periapical radiographs. Periapical radiographs taken at the time of implant placement were compared to periapical radiographs taken at the current maintenance appointment to assess crestal bone loss. Width of keratinized gingiva was divided into two groups using a $2 \mathrm{~mm}$ cut-off. Group 1 was defined a simplants. where the width of the surrounding keratinized gingiva was $2 \mathrm{~mm}$ or greater; Group 2 defined implants where the width of the surrounding keratinized gingiva was less than $2 \mathrm{~mm} .^{2}$ 


\section{Results}

Sixty-nine patients were studied $(\mathrm{n}=69)$. Thirty-six patients (52.2\%) were $26-50$ years old and 33 patients $(47.8 \%)$ ranged in age from $51-75$ years. Forty-nine patients $(71.0 \%)$ were male, while 20 $(29.0 \%)$ were female. Thirty-four $(49.3 \%)$ patients reported current use of tobacco products, while $35(50.7 \%)$ denied use of tobacco. Eighteen patients $(26.1 \%)$ had keratinized gingiva less than $2 \mathrm{~mm}$ surrounding their implants, compared to 51 patients $(73.9 \%)$ who had more than $2 \mathrm{~mm}$ of keratinized gingiva surrounding their implants. Bleeding upon probing was seen in 17 patients $(24.6 \%)$ and was absent in 52 patients $(75.4 \%)$. Pocket depths of more than $3 \mathrm{~mm}$ were noted in 22 patients $(31.9 \%)$, compared to pockets depths less than $3 \mathrm{~mm}$ noted in 47 patients $(68.1 \%)$ (Table 1$)$.

Table I Keratinized gingiva research case report

\begin{tabular}{|c|c|}
\hline Characteristics & Number of patients \\
\hline Age & 0 \\
\hline$<25$ years & 36 \\
\hline $26-50$ years & 33 \\
\hline $5 \mathrm{I}-75$ years & 0 \\
\hline \multicolumn{2}{|l|}{ Gender } \\
\hline Male & 49 \\
\hline Female & 20 \\
\hline \multicolumn{2}{|l|}{ Smoke } \\
\hline Yes & 34 \\
\hline No & 35 \\
\hline \multicolumn{2}{|l|}{ KG Levels } \\
\hline$<2 \mathrm{~mm}$ & 18 \\
\hline$>2 \mathrm{~mm}$ & 51 \\
\hline \multicolumn{2}{|l|}{ Bleeding } \\
\hline Yes & 17 \\
\hline No & 52 \\
\hline \multicolumn{2}{|l|}{ Redness } \\
\hline Yes & 3 \\
\hline No & 66 \\
\hline \multicolumn{2}{|l|}{ Pocket Depth } \\
\hline$>3 \mathrm{~mm}$ & 22 \\
\hline$<3 \mathrm{~mm}$ & 47 \\
\hline \multicolumn{2}{|l|}{ Timing of Implant } \\
\hline$<2$ Years & 5 \\
\hline 2-3 Years & 15 \\
\hline$>3$ Years & 49 \\
\hline
\end{tabular}

The data was analyzed using a chi square test to determine if the width of keratinized gingiva significantly affected probing depths and bleeding upon probing. The data was considered statistically significant if the computed $\mathrm{p}$ value was less than or equal to 0.05 . Multivariate regression analysis was done to determine whether the width of keratinized gingiva was independently associated with bleeding upon probing. Smoking and gender were adjusted. Age was not distributed well enough to be used in the model. Other variables were not significant and were not included in the final model. Chi square analysis showed an association between amount of keratinized gingiva and pocket depths. Statistical analysis of the data for pocket depths showed no significant association between pocketing and amount of keratinized gingiva; indicating that a lack of keratinized gingiva does not result in greater pocket depths $(\mathrm{p}=0.878)$ (Table 2$)$.

Table 2 Pocketing vs keratinized gingiva

\begin{tabular}{llll}
\hline Pocketing $>3 \mathbf{m m}$ & \multicolumn{2}{l}{ KG $\mathbf{2 m m}$} & \multicolumn{2}{c}{ Total } \\
\cline { 2 - 3 } & Yes & No & \\
\hline Yes & 6 & 16 & 22 \\
& 27.27 & 72.73 & 100 \\
& 33.33 & 31.37 & 31.88 \\
No & 12 & 35 & 47 \\
& 25.53 & 74.47 & 100 \\
& 66.67 & 68.63 & 68.12 \\
Total & 18 & 51 & 69 \\
& 26.09 & 73.91 & 100 \\
& 100 & 100 & 100 \\
\hline
\end{tabular}

Chi square $=0.0236 \mathrm{P}=0.878$ Statistical analysis of the data for bleeding upon probing showed a statistically significant association between the amount of keratinized gingiva and bleeding upon probing $(\mathrm{p}=0.023)$. Eighty-two percent of implants with less than $2 \mathrm{~mm}$ keratinized gingiva experienced bleeding upon probing, compared to $56 \%$ of implants with keratinized gingiva greater than $2 \mathrm{~mm}$. (Table 3) Logistic regression was performed to adjust for smoking and gender. Since age was not distributed well enough, it was not used in the model. Variables such as suppuration, mobility of the implant, timing of implant placement, and width of the implant were not significant and not represented in the final model. Table 4 illustrates that implants with less than $2 \mathrm{~mm}$ keratinized gingiva are 6.5 times more likely to experience bleeding upon probing than those implants with greater than $2 \mathrm{~mm}$ of keratinized gingiva $(\mathrm{OR}=6.5)$. The final model was adjusted for smoking and gender.

Table 3 Bleeding vs keratinized gingiva

\begin{tabular}{llll}
\hline \multirow{2}{*}{ Bleeding } & KG $\mathbf{2 m m}$ & \multicolumn{2}{c}{ Total } \\
\cline { 2 - 3 } & Yes & No & \\
\hline Yes & 8 & 9 & 17 \\
& 47.06 & 52.94 & 100 \\
No & 44.44 & 17.65 & 24.64 \\
& 10 & 42 & 52 \\
& 19.23 & 80.77 & 100 \\
Total & 55.56 & 82.35 & 75.36 \\
& 18 & 51 & 69 \\
& 26.09 & 73.91 & 100 \\
& 100 & 100 & 100 \\
\hline
\end{tabular}


Table 4 Regression final model

\begin{tabular}{lllllll}
\hline Bleeding & Odds ratio & Standard error & $\mathbf{Z}$ & $\mathbf{P}>|\mathbf{z}|$ & $\mathbf{9 5 \%} \mathbf{C l}$ & \\
\hline KG $<2 \mathrm{~mm}$ & 6.483607 & 4.868434 & 2.49 & 0.013 & 1.488 & 28.25 \\
Gender & 2.780876 & 2.123517 & 1.34 & 0.18 & 0.623 & 15.42 \\
Smoking & 12.19472 & 9.778232 & 3.12 & 0.002 & 2.533 & $58.7 \mid$ \\
Cons & 0.246288 & 0.1948526 & -1.77 & 0.077 & 0.521 & 1.162 \\
\hline
\end{tabular}

\section{Discussion}

Several studies have suggested that a minimal width of keratinized gingiva around dental implants is necessary for the continued health and stability of the implant, while other studies have failed to demonstrate any need for minimal width. Results of this study suggest that implants surrounded by less than $2 \mathrm{~mm}$ keratinized gingiva have an increased amount of bleeding upon probing, which is a clinical indication of active inflammation. Prolonged inflammation around dental implants can result in subsequent attachment apparatus loss as well as bone loss, ultimately leading to failure of the implant. The standard laid out by Lang, et al., ${ }^{3}$ asserts that the minimum width for healthy keratinized tissue surrounding the teeth is $2 \mathrm{~mm}$. The standard is routinely extended to peri-implant keratinized tissue as well. Research has challenged this concept in relationship to teeth and implants, asserting the opposing viewpoint, that a minimum width of keratinized gingiva is not required around teeth or implants, provided adequate oral hygiene is maintained. Cox and Zarb et al., ${ }^{4}$ in 1987 found that $80 \%$ of evaluated implants had no keratinized gingiva but had healthy peri-implant tissue. Similarly, Esper et al., 5 showed no statistically significant difference between bleeding upon probing and plaque control and width of keratinized gingiva. Proper oral hygiene may be better facilitated in areas of adequate keratinized gingiva. ${ }^{6}$

Healthy keratinized gingiva around dental implants results in more predictable success and maintenance of the implant, and also results in an improved esthetic outcome. Keratinized gingiva provides stabilization to the periodontium, protects the teeth and implants from masticatory and external trauma, and provides a barrier to inflammatory infiltrate. ${ }^{7}$ while, the sample size in this study is limited, we feel that implants should have a minimum amount of $2 \mathrm{~mm}$ of keratinized gingiva to maintain health. We believe that reconstruction of the keratinized gingiva in deficient areas using techniques such as the free gingival graft or the sub epithelial connective tissue graft should be employed prior to implant placement.

\section{Conclusion}

Despite limited data in this study, we concluded that regardless of the amount of keratinized gingiva present, implant placement among the subjects was successful. Implants with less than $2 \mathrm{~mm}$ keratinized gingiva exhibited clinical signs of inflammation in increased bleeding upon probing and redness. The caveat remains: Persistent inflammation around an implant may contribute to later failure. The findings of this research support gingival augmentation prior to implant placement to prevent future failure where minimal keratinized gingiva exists. Further studies may be needed to confirm the findings from this study due to its small sample size.

\section{Acknowledgments}

None

\section{Conflicts of interest}

The author declares that there are no conflicts of interest.

\section{Funding}

None

\section{References}

1. Chung DM, Oh TJ, Shotwell JL, et al. Significance of keratinized mucosa in maintenance of dental implants with different surfaces. J Periodontol. 2006;77(8):1410-1420.

2. Adibrad M, Shahabuei M, Sahabi M. Significance of the width of keratinized mucosa on the health status of the supporting tissue around implants supporting overdentures. J Oral Implantol. 2009;35(5):232-237.

3. Lang NP, Loe H. The relationship between the width of keratinized gingiva and gingival health. J Periodontol. 1972;43(10):623-627.

4. Cox JF, Zarb GA. The longitudinal clinical efficacy of osseointegrated dental implants: a 3-year report. Int $J$ Oral Maxillofac Implants. 1987;2(2):91-100.

5. Esper LA, Ferreira SB, Kaizer Rde O, et al. The role of keratinized mucosa in peri-implant health. Cleft Palate Craniofac J. 2012;49(2):167-170.

6. Salvi GE, Lang NP. Diagnostic parameters for monitoring peri-implant conditions. Int J Oral Maxillofac Implants. 2004;19(1):116-127.

7. Paiva RBM, Mendonca JAG, Zenobio EG. Peri-implant tissues health and its association to the gingival phenotype. Dental Press Implantology. 2012;6(4):104-113. 